\title{
ON TWO NANOCOMPOSITE MODELS: DIFFERENCES, SIMILARITIES AND INTERPRETATIONAL POSSIBILITIES REGARDING TSAGAROPOULOS' MODEL AND TANAKA'S MODEL
}

\author{
Michael G. Danikas *
}

\begin{abstract}
Nanocomposites consist nowadays a major field of research. This paper investigates the interpretational possibilities of two models developed, namely, Tsagaropoulos' model and Tanaka's model. The two models were developed separately and independently from each other. They both consider various layers around the nanoparticles. They both consider well bonded and more loosen layers. Similarities and differences between the two models are pointed out as well as possibilities for further research in order to shed light on some aspects of electrical phenomena in these materials.

K e y w o r ds: nanocomposites, nanoparticles, glass transition temperature, electrical trees, dielectric breakdown, discharge path
\end{abstract}

\section{INTRODUCTION}

There are several years now that researchers try to improve conventional polymers by adding minute particles (nanoparticles) into the polymer matrix, changing thus their electrical, mechanical and thermal properties [1]. There is already a vast body of technical literature regarding nanocomposites and their related properties [2].

Of particular interest is the subject as to why nanoparticles inserted into conventional polymers improve so dramatically the various properties of nanocomposites. Two of the most interesting models that have been developed wrt the interpretation of some of the electrical properties and/or electrical phenomena in nanocomposites are the following: Tsagaropoulos' model [3] was proposed especially as a possible explanation as to why nanocomposites show the behavior they do, regarding the glass transition temperature. Tanaka's model was proposed as a possibility to explain various phenomena, such as electrical treeing and dielectric breakdown in nanocomposites $[4,5]$.

It is the aim of the present paper to analyze aspects of these two models as well as to compare them regarding their interpretational possibilities.

\section{MODEL OF TSAGAROPOULOS}

Tsagaropoulos' model is analyzed in [3]. It assumes two basic layers around a nanoparticle, an inner tightly bound layer in which polymer motion is severely restricted, and another intermediate layer which is more loosely bound. The first layer - at a distance of 1 to 20 Angstrom from the nanoparticle surface, the polymer is physically (if not chemically) adsorbed and its dynamics is slowed down as a consequence of this - is generally thought to be tightly bound. At larger distances of about 25 to 90 Angstrom from the nanoparticle surface, the polymer is believed to be less tight and perform an intermediate dynamics; this layer is more loosely bound [6]. In [7], a third layer is also assumed, that of the unrestricted bulk polymer. In Fig. 1, the morphology of the polymer around filler particles is shown. It shows, in other words, the sequence of events accompanying the incorporation of increasing amounts of silica particles in the polymer. This results in a decrease of the average interparticle distance d. Layers of polymer, shown in black in Fig. 1, are tightly bound to nanoparticles. The model deals in principle with the two glass transition temperatures in nanofilled polymers. The polymer chains capable of participating in the second glass transition will be called polymer of reduced mobility or loosely bound polymer. In [3], the critical percentage of nanoparticles is put up to $10 \%$, probably because it is at this percentage where the average interparticle distance has a 'critical' value $\mathrm{d}_{c r}$. As the average interparticle distance decreases further, with the incorporation of more filler particles, the mobility restrictions become so severe that the loosely bound polymer is gradually transformed to tightly bound (Fig. 1C). The volume fraction of loosely bound polymer decreases, resulting in a decrease in the area of the second $\tan \delta$ peak. On the contrary, the volume fraction of tightly bound polymer increases. As the interparticle distance decreases, it is the most restricted regions of loosely bound polymer which are transformed first into tightly bound polymer. The regions with the highest $\mathrm{Tg}$ will be eliminated earliest as filler content increases, resulting in an actual decrease of the second Tg. At lower filler content, the amount of the immobilized polymer around the particles is small, allow-

* Democritus University of Thrace, Department of Electrical and Computer Engineering, Xanthi, Greece, mdanikas@ee.duth.gr 


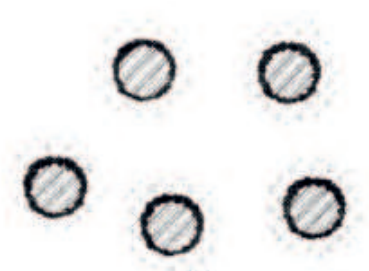

(a)

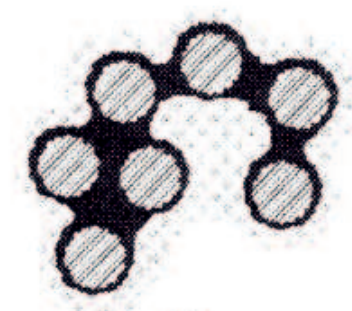

(c)

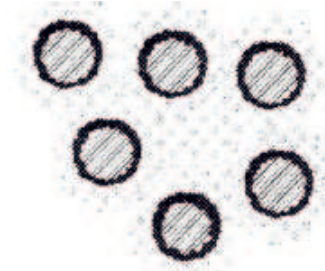

(b)

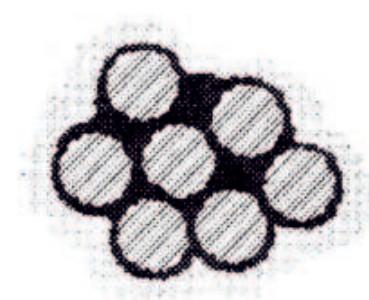

(d)

Fig. 1. Schematic model of the morphological transformations in filled polymers, occurring as the content increases from less than $10 w t \%(\mathrm{~A})$, to about $10 w t \%$ (B), to more than $20 w t \%(\mathrm{C})$ and to over than $50 w t \%$ (D). The line-shaded areas are the silica particles, whereas the black areas correspond to tightly bound polymer and the gray areas to rather loosely bound polymer (after [3]).

ing for sufficiently strong interactions between the loosely bound polymer and the particles. As the amount of immobile polymer increases at higher nanoparticle contents, such interactions will become weaker. Consequently, as the immobile polymer does not restrict the chain mobility as efficiently as the nanoparticles, the second $\mathrm{Tg}$ decreases. Eventually, with a very high nanoparticle content (in [3], the author talks of contents of more than $50 w t \%$ ), the average interparticle distance will be much smaller than $\mathrm{d}_{c r}$, with the result that nearly all polymer chains are immobilized.

Higher filler loading implies smaller interparticle distances, the volume fraction of the loose polymer layer reduces and the nanoparticles themselves start acting as barriers to the flow of current between the electrodes [8]. The focus of Tsagaropoulos' model is on the influence of filler particles on the mobility of polymer chains [9]. Tsagaropoulos' model claims that the discrete bound layer can result in a second glass transition temperature but work on thin films did not point to a discrete layer of affected material but to a far field effect [10]. To emphasize this point, in yet another publication, it was remarked that the two-layer model by Tsagaropoulos does not embody the physics of the whole situation [11]. In the latter publication, it was claimed that the mere presence of regions of modified mobility in the proximity of nanoparticles cannot explain the glass transition temperature results. The smallest interparticle spacings, which would percolate first, would dominate the behavior. Such interaction effects apply also in the case of funtionalized nanoparticles, the only difference being that in close vicinity to the nanoparticles, the regions have reduced mobility. The glass transition process in restricted geometries requires the interaction of near surface regions of

altered mobility. Although the authors of [11] differentiate from Tsagaropoulos' model, they also use terms such as 'regions of reduced mobility', which remind us well of Tsagaropoulos' model.

Some justification of Tsagaropoulos' model is mentioned in [7], where NMR data support it, since as was mentioned in a previous paper, ' $\ldots$ the adsorption layer differing in mobility from bulk PDMS could be detected just above the glass transition temperature in systems filled with hydrophobic Aerosil' [12]. Further support for this model was given in [6], where faster processes correspond to bulk polymer whereas slower processes are interpreted in terms of tightly bound polymer chains, whose segmental dynamics are slowed down wrt bulk dynamics as a consequence of the interaction with the silica nanoparticle surface. Further support is offered in [13], where Dynamic Mechanical Thermal Analysis suggests that polymer chain motion in polymer electrolytes is affected by the presence of nanoparticle surfaces, ie glass transition temperature increases by increasing the percentage of nanoparticles.

Further support for Tsagaropoulos' model can also be traced in [14], where the broadening of the $\tan \delta$ peak to higher temperatures as well as the decrease of the magnitude of $\tan \delta$ peak are attributed, by the authors, to the variation in interaction between PAN (polyacrylnitrile) and ATO (antimony-doped tin oxide). The motion of PAN molecules closer to the nano-ATO particles would be more constrained than those farther away from it. Echoes of Tsagaropoulos' model can also be found in [15], where with a high fraction of nanoparticles offers a lower degree of crystallization might occur, since the mobility of the polymer matrix chain segments will decrease during the period of crystallization. In [16], the authors seem to accept Tsagaropoulos' model claiming that 'for the $\mathrm{PC} / \mathrm{MWNT}$ composite, the higher $\tan \delta$ peak becomes broader, which may be considered that the mobility of the polymer chain becomes more restricted by the MWNT ... [possible explanation is that] ... the phase separation morphology model according [to which] the lower tan delta peak of the polymer composites corresponded to the $\mathrm{Tg}$ of the polymer matrix, and the higher $\tan \delta$ peak of the polymer composites corresponded to the $\mathrm{Tg}$ of the confined polymer chain by the filler'. Also in [17], a second thermal transition above the glass transition which was noted for samples in excess of $20 w t \%$ fumed silica, was attributed to the polymer closely interacting with the silica filler, ie what is termed bound polymer. Recently, it was pointed out that nanofillers can constrain the mobility of polymer chains as well as their relaxation spectra, which can change the glass transition temperature and modulus of the matrix [18].

As a general comment, one may conclude that Tsagaropoulos' model, although subjected to some criticism already mentioned, is generally accepted as a model explaining various aspects of nanocomposite behavior, especially those having to do with the glass transition temperature. 


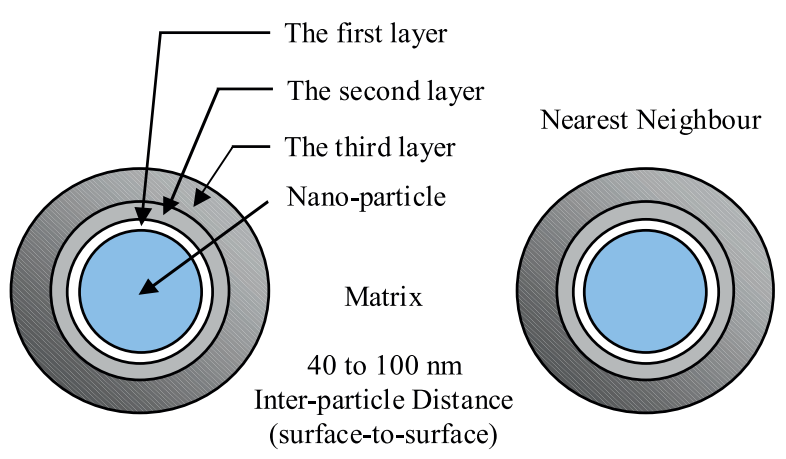

Fig. 2. Outline of the multi-core model for polymer nanocomposites (after [4])

\section{MODEL OF TANAKA}

Tanaka's model consists of a multicore description for nanoparticles $[4,5]$. It bears also the name 'multi-core' model since it considers more than two layers. Tanaka working independently of Tsagropoulos, proposed another model. There is a bonded layer around the nanoparticle (first or innermost layer), a bound layer (second layer), a loose layer (third layer) and an electric double layer overlapping the above three layers. A picture of Tanaka's model is given in Fig. 2. The first layer corresponds to a transition layer tightly bonded to both inorganic and organic substances by coupling agents, such as silane. Its thickness is postulated to be of about $1 \mathrm{~nm}$. The first layer is tied by ionic, covalent, hydrogen and van der Waals bondings. The second layer is an interfacial region consisting of a layer of polymer chains strongly bound and/or interacted to be first layer and the surface of the inorganic particle. The thickness of this layer is between 2 and $9 \mathrm{~nm}$. These values depend on the strength of the polymer-particle interaction. Obviously, the stronger the interaction, the larger the polymer fraction. Chain mobility and crystallinity are deeply involved. The former is directly related to glass transition temperatures. The average chain conformation can be altered and/or the type and degree of crystallinity can change. The third layer is a region of loosely coupling and interacting to the second layer. The loose layer has different chain conformation, chain mobility and even free volume or crystallinity from the polymer matrix. This layer is of several tens of nm thick. The fourth layer, the electric double layer, forms a long distance dipole. This will affect electrical conduction and dielectric properties in the low frequency region. As is indicated in [5], a nanoparticle is charged positively or negatively when a polymer has mobile charge carriers, which are distributed in the interface so that the counter charges with the opposite polarity are diffused outward from the contact surface to the Debye shielding length corresponding to the Couy-Chapman diffuse layer in which charge decays exponentially according to the Born approximation. In other words, Tanaka's model considers nanoparticles are positively or negatively charged, taking into account that are inserted into polymeric materials which end to be either positively or negatively charged. Such charging may in some ways have an effect on discharge treeing paths. Simulation results until now give some good grounds to Tanaka's model. Figures 3 and 4 show the electrical tree propagation in a nanocomposite, and it is evident that the tree paths go through the polymer and not through the nanoparticles. Treeing paths may propagate through the polymer and/or they may touch nanoparticles and circumvent them. Simulation data show that in no case treeing paths go through the nanoparticles. Although one may say that these are just simulation results, ie they do not take into account all possible involved parameters, it is undeniable that they at least — partially simulate realistic conditions. Moreover, simulations with homocharge presence show that the tree propagation stops at a certain stage. This although it may not explain the gist of Tanaka's model, it shows, however, that with charges of the same polarity the tree growth stops.

Tanaka's model was extensively discussed in [19], where it was remarked that the third layer will have a dominant effect on the overall performance of the nanocomposite, as being much larger than the first two layers. With high nanoparticle percentage, about $50 \%$ of the total volume will be affected and one may assume that it is possible that also the shell layer interfaces will overlap. This point is somehow shown, albeit with all reservations of the simulation parameters, in Figs. 3 and 4 , where treeing paths penetrate through the polymer. In the aforementioned figures, the tree propagation is shown in a nanocomposite with well defined nanoparticles and a space charge density of $40 \mathrm{C} / \mathrm{m}^{3}$.

Homocharge of $40 \mathrm{C} / \mathrm{m}^{3}$ is assumed. The distance between the electrodes is $0.4 \mathrm{~mm}$, a $5000 \times 5000$ grid was used, the dimensions of the nanoparticles are $100 \mathrm{~nm}$ (each side is $100 \mathrm{~nm}$ ), the distance between the nanoparticles was $300 \mathrm{~nm}$ (from centre to centre).

The tree propagation stopped with homocharges present. Tanaka's model was discussed in [20-22], where the authors used an analogy of the model to explain the percolation of charge carriers through overlapping water shells. In [20-22], it was claimed that the inner layer of water may surround the nanoparticle and the second water layer to be dispersed but sufficiently concentrated to be conductive. It was considered that the inner and outer layers are likely to provide a channel for charges and carriers. Once the water shell around a nanoparticle can overlap with the others, charges and carriers may move with through the interaction zone between neighboring particles. Tanaka's model in fact, in the above publications, helped to study the electrical properties of epoxy resin nanocomposites as a function of hydration.

In yet another publication, the authors tend to agree with Tanaka's model and with Tanaka's estimate of the interaction zone as a fraction of a $\mathrm{nm}$ when the coupling 


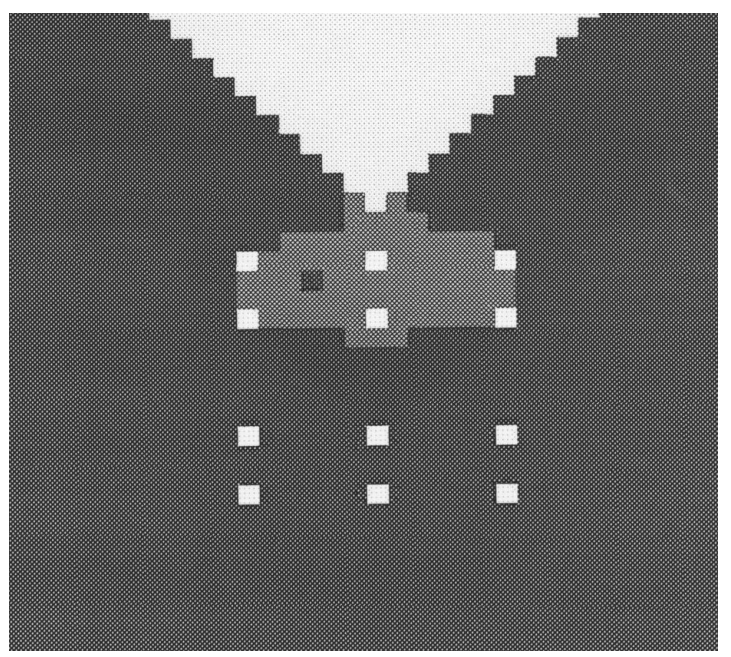

Fig. 3. Simulation in a point/plane electrode arrangement of electrical tree propagation. The dielectric constant of the polymer (in black colour) is assumed to be 4, the dielectric constant of the nanoparticles (in white colour) is assumed to be 8 , the area of the tree propagation is depicted in grey colour.

of inorganic surface with the polymer matrix increases by using silane [23]. The authors of [23], however, put more emphasis on the intensity and specificity of the chemical activity — which will affect the polymerization of the interface - and not so much on the thickness of this zone. Another researcher, in [24], seems to accept Tanaka's model when he discusses overlapping zones between the nanoparticles and complete percolation through interaction zones and volumetric concentration. In the same publication, he carries on to ask pertinent questions regarding the nature of interaction zone around the nanoparticles, the layers which comprise the interaction zones and their thicknesses.

Tanaka's model is much more recent than Tsagaropoulos's model. It was positively received by the scientific community since it offers plausible explanations as to the some aspects of the electrical behavior of nanocomposites. It must, however, be noted that more experimental results, and particularly photographic evidence, are needed in order to fully verify it. Optical microscopy as well as SEM are needed to exactly trace the discharge and/or treeing paths in nanocomposites. This point is also stressed below.

\section{SIMILARITIES AND DIFFERENCES BETWEEN THE TWO MODELS}

Regarding interpretational possibilities, Tanaka's model is not different from Tsagaropoulos' model. Both models tend to assume an inner bonding layer, strongly attached to the nanoparticle, they differ, however, somehow (but not radically) in the way they perceive the reaction to other nanoparticles and/or to the rest of the polymer. Their major difference is in the number of assumed layers, and not so much as to what these layers may do. Tanaka's model seems to be more complete in that it

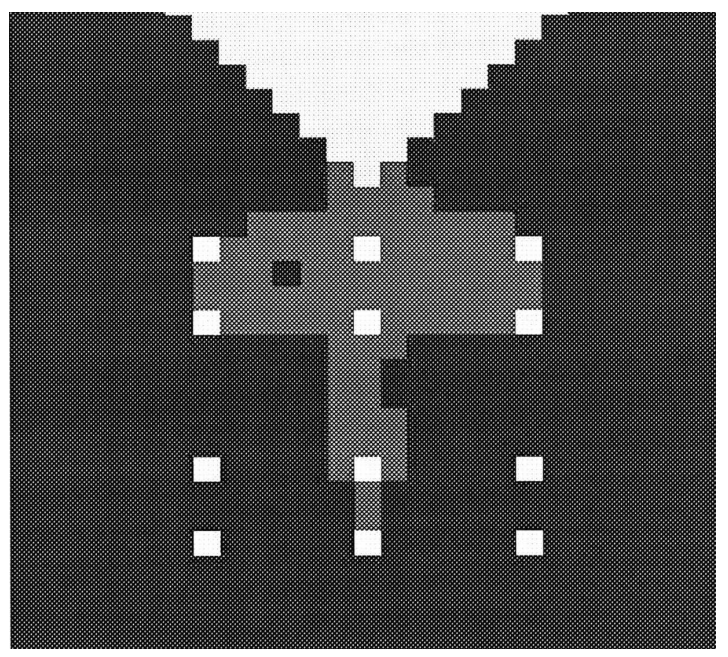

Fig. 4. Simulation in a point/plane electrode arrangement of electrical tree propagation. Fig. 4 is the continuation of the simulation in Fig. 3. The data were as in Fig. 3, the only difference being that the tree propagation advanced but eventually stopped. Tree propagation did not progress to the opposite electrode.

considers in some detail the workings of the polymer matrix/nanoparticle interface. Both models try to explain the second glass transition temperature. Both models assume that the interparticle region is the weak link in the polymer — nanoparticle system. Tsagaropoulos' model tends to explain rather well the glass transition temperature variation when nanoparticles are added to a conventional polymer. Tanaka's model also tries to explain the glass transition temperature variations by claiming that '... improvement in glass transition temperature must originate from the formation of the second and third layers of the multi-core model. It indicates that fillers and matrices are rather tightly bonded through these layers' $[4]$.

Tsagaropoulos' model refers more to glass transition temperature data without any elaboration regarding the electrical treeing and dielectric strength of nanocomposites. Tanaka's model tackles precisely these issues. The two aforementioned models differ in their number of layers, their respective thicknesses, their possibility of interpretation of various phenomena. Still, they both refer to layers surrounding a nanoparticle and they both explain why with the addition of nanoparticles there is a marked improvement in the properties of conventional polymers. Recent simulation results (Figs. 3 and 4 ) indicate that electrical treeing goes through the polymer matrix, avoiding thus the nanoparticles. This is an indication of validity of both models but not yet a proof. The indication seems to be stronger in Tanaka's model (which assumes charged nanoparticles), since the simulations show that - in the presence of homocharges - trees propagate through the polymer matrix. One may well argue that homocharges are not the same and they are not perceived as the same as the charged nanoparticles in Tanaka' model. Tanaka's model assumes an electric double layer for each nanoparticle, and it also assumes that this is the result of triboelectricity. The answer to such claims is that, although 
homocharges are something different, they also assume the same polarity charges around the nanoparticles and in this respect, their end result will be the same. These simulation data, however, do not go against Tsagaropoulos' model since this model is also based on tightly bound layers hindering breakdown events and thus strengthening the nanocomposite.

It has to be clear that the above two models are not by any means exhausted in the references cited here. In the context of this work, we tried to approach both models, their respective merits and aspects in need of further research, but it was not possible to include all publications which referred to these two models. That would be an immense task and, in all probability, beyond the scope of a journal paper.

\section{SOME THOUGHTS AND PROPOSALS FOR FURTHER RESEARCH}

What is missing for the time being, is not so much experimental data regarding the improvement of the electrical properties of nanocomposites wrt the conventional composites, but more photographic evidence. In this respect, SEM photographs, among others, may indeed give information as to the directions followed by treeing and/or discharge paths in nanocomposites. They may indicate whether such paths avoid the nanoparticles. SEM evidence for the time being confirms that nanoparticles act as a sort of barriers opposed to the advancement of electrical treeing. SEM evidence confirms that discharge and treeing paths go round the nanoparticles. More evidence, however, is needed.

Physical models regarding the treeing path have been proposed [25]. Such models propose that trees will dwell at the nanoparticle surface and try to propagate through the nanoparticle-polymer interface. The nanoparticles would create a hindrance to growth, and they will prevent trees to grow in rather straightforward directions. Trees will propagate from nanoparticle to nanoparticle through the polymer. Consequently, the tree structure will be dense and zigzag. Such models, however, no matter how plausible explanations they may offer, have to be supported by evidence and this evidence can be given by sophisticated photographic techniques.

Needless to say that, the two aforementioned models have to be tried to various kinds of nanocomposites. It is only through the variety of combinations nanoparticlepolymer that more insight as to the workings of the improvement of the nanocomposite properties may be gained.

\section{CONCLUSIONS}

In this paper, a comparison between two well known models is being made. Both models have their own merit, both of them are supported by some experimental evidence and recent simulation data. The models,
Tsagaropoulos' model and Tanaka' s model, have their own explanatory power and they are based on the idea of more and less well bonded layers around nanoparticles in the polymer matrix. More work has to be carried out, however, regarding the photographic evidence of the treeing and/or discharge paths in nanocomposites.

\section{Acknowledgments}

The author wishes to express his gratitude to Professor Toshikatsu Tanaka of Waseda University, Graduate School of Information, Production and Systems, for having invited him as Visiting Scholar in his laboratory during the academic year 2008-09 and having introduced him to the subject of nanocomposites. He also wishes to thank Miss D. Pitsa for her simulation results on electrical tree propagation in nanocomposites.

\section{REFERENCES}

[1] LEWIS, T. J. : Nanometric dielectrics, IEEE Trans. Diel. Electr. Insul 1 (1994), 812-825.

[2] See for example the whole issue of IEEE Trans. Diel. Electr. Insul. 15 No. 1 (Feb 2008).

[3] TSAGAropoulos, G.-EISEnBERG, A.: Dynamic Mechanical Study of the Factors Affecting the Two Glass Transition Behavior of Filled Polymers. Similarities and Differences with Random Ionomers, Macromolecules 28 (1995), 6067-6077.

[4] TANAKA, T.: 'Dielectric Nanocomposites with Insulating Properties, IEEE Trans. Diel. Electr. Insul. 12 No. 5 (2005), 914-928.

[5] TANAKA, T. Polymer Nanocomposites as HV Insulation: Superiority and Expectation: Proc. XVth Int. Symp. High Volt. Eng., Ljubljana, Slovenia, Aug 27-31, 2007.

[6] TRIOLO, A.-LO CELSO, E.-NEGRONI, F.-ARRIGHI, V.-QIAN, H.-LECHNER, R. E.-LECHNER, A.-DESMEDT, A.-PIEPER, J.-FRICK, B.-TRIOLO, R. : QENS Investigations of Filled Rubbers, Appl. Phys. A: Mater. Sci. and Process. 74 (2002), 490-492.

[7] ARRIGHI, V.-McEWEN, I. J.-QIAN, H.-SERRANO PRIETO, M. B.: The Glass Transition and Interfacial Layer in Styrene-Butadiene Rubber Containing Silica Nanofiller, Polymer 44 No. 20 (2003), 6259-6266.

[8] SINGHA, S.-JOY THOMAS, M.: Dielectric Properties of Epoxy Resin Nanocomposites, IEEE Trans. Diel. Electr. Insul. 15 No. 12 (008).

[9] BERGLUND, L. A. : Reology of Polymer Nanocomposites Are There Unique Effects for Exploitation?, Annual Transactions of the Nordic Rheology Society 14 (2006).

10] AJAYAN, P. M.-SCHADLER, L. S.-BRAUN, P. V.: Nanocomposite Science and Technology, Eds. Wiley-VCH Verlag, Weinheim, Germany, 2003.

[11] BANSAL, A.-YANG, H.-LI, C.-CHO, K.-BENICEWICZ, B. C.-KUMAR, S. K.-SCHADLER, L. S.: Quantitative Equivalence between Polymer Nanocomposites and Thin Polymer Films, Nature Mater. 4 (2005), 693-698.

[12] LITVINOV, V. M.-SPIESS, H. W. H NMR Study of Molecular Motions in Polydimethylsiloxane and its Mixtures with Aerosils : Makromol. Chem. 192 No. 12 (1991), 3005-3019.

[13] KERR, J. B.-HAN, Y. B.-LIU, G.-REEDER, C.-XIE, J.-SUN, X. : Interfacial Behavior of Polymer Electrolytes, Lawrence Berkley National Laboratory, Paper LBNL-53141, 2003. 
[14] WEI PAN-HANDAO ZOU: Characterization of PAN/ATO Nanocomposites Prepared by Solution Blending, Bul. Mater. Sci. 31 No. 5 (2008), 807-811.

[15] KUO, M. C.-TSAI, L. M.-HUANG, J. C.-CHEN, M. : PEEK Composites Reinforced by Nano-Sized $\mathrm{SiO} 2$ and $\mathrm{Al} 2 \mathrm{O} 3$ Particulates, Mater. Chem. And Phys. 90 (2005), 185-195.

[16] SUNG, Y. T.-KUM, C. K.-LEE, H. S.-BYON, N. S.YOON, H. G.-KIM, W. N. : Dynamic Mechanical and Morphological Properties of Polycarbonate/Multi-Walled Carbon Nanotube Composites, Polymer 46 No. 15 (2005), 5656-5661.

[17] BURNSIDE, S. D.-GIANNELIS, E. P.: Nanostrusture and Properties of Polysiloxane-Layered Silicate Nanocomposites, J. Polym. Sci. Part B: Polym. Phys. 38 No. 12 (2000), 1595-1604.

[18] SAMAI, S. S.: Role of Temperature and Carbon Nanotube Reinforcement on Epoxy based Nanocomposites, J. Miner. \& Mater. Charact. \& Eng. 8 No. 1 (2009), 25-36.

[19] HAJIYANNIS, A.-CHEN, G.: Space Charge Formation in Epoxy Resin Including Various Nanofillers, 2008 Ann. Rep. CEIDP, pp. 714-717.

[20] CHEN ZOU-FOTHERGILL, J. G.-ROWE, S. W.: The Effect of Water Absorption on the Dielectric Properties of Epoxy Nanocomposites, IEEE Trans. Diel. Electr. Insul. 15 No. 1 (2008), 106-117.

[21] ZOU, C.-FU, M.-FOThERGILL, J. C.-ROWE, S. W. : The Influence of Water on Dielectric Behavior of Silica-Filled Epoxy Nano-Composites and Percolation Phenomena, 2007 Ann. Rep. Conf. Electr. Insul. Diel. Phen.
22] ZOU, C.-FOTHERGILLL, J.-ROWE, S.: A 'Water Shell' Model for the Dielectric Properties of Hydrated Silica Filled Epoxy Nano-Composites, 2007 Ann. Rep. Conf. Electr. Insul. Diel. Phen.

23] FRECHETTE, M. F.-REED, C. W. : 'The Role of Molecular Dielectrics in Shaping the Interface of Polymer Nanodielectrics, 2007 Ann. Rep. Conf. Electr. Insul. Phen..

24] FOTHERGILL, J. C.: Ageing, Space Charge and Nanodielectrics: Ten Things We Don't Know about Dielectrics, 2007 Int. Cof. Solid Diel., Winchester, UK, July 8-13, 2007, pp. 1-10.

[25] AlAPATI, S.-JOY THOMAS, M.: 'Electrical Treeing in Polymer Nanocomposites, $15^{\text {th }}$ National Power Systems Conference (NPSC), IIT Bombay, December 2008, pp. 352-355.

Received 19 June 2009

Michael G. Danikas is Associate Professor with the Department of Electrical and Computer Engineering, Democritus University of Thrace (DUTh). He was with Eindhoven University of Technology, The Netherlands, and with ABB, Baden-Daettwil, Switzerland, before he joined DUTh. His research interests include breakdown in transformer oil, polymeric insulation breakdown processes, vacuum breakdown, partial discharge activity at inception voltages, simulation of electrical trees in polymeric materials and nanocomposites and partial discharges in rotating machine insulation.

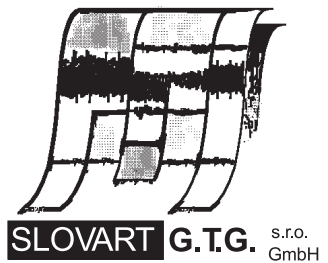

EXPORT - IMPORT
EXPORT - IMPORT

of periodicals and of non-periodically printed matters, books and CD-ROMs

Krupinská 4 PO BOX 152, 85299 Bratislava 5, Slovakia tel: ++421 263839 472-3, fax: ++421 263839485 info@slovart-gtg.sk; http://www.slovart-gtg.sk

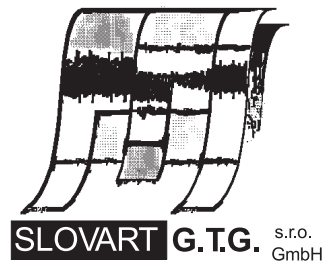

EXPORT - IMPORT 